\title{
Psychological Spectrum Experienced by Heart Failure Patients After Left Ventricular Assist Device Implantation
}

\author{
Nkechi A. Okam ${ }^{1}$, Wiqas Ahmad ${ }^{1}$, Dibyata Rana ${ }^{1}$, Chenet Torrilus ${ }^{1}$, Nusrat Jahan ${ }^{1}$, Surik Sedrakyan ${ }^{1}$ \\ 1. Internal Medicine, California Institute of Behavioral Neurosciences \& Psychology, Fairfield, USA
}

Corresponding author: Nkechi A. Okam, kech_alina@yahoo.com

\begin{abstract}
Depression and anxiety disorders are prevalent in patients with heart failure. They are associated with adverse effects such as rapid disease progression, poor medication compliance, low quality of life and increased mortality rate. The current literature review aims to provide an overview of the overall rate of depression in patients who receive left ventricular assist device (LVAD) implantation and identify the psychological phases that these individuals experienced peri- and post-LVAD implantation. A PubMed search using regular and Medical Subject Headings (MeSH) keywords identified 239 articles. After applying inclusion/exclusion criteria, removal of duplicate studies, and careful review of articles, 40 studies provided relevant information on our primary end-point. These 40 studies selected include 13 paid articles with abstracts and 27 free full-text articles comprising eight prospective cohort studies, five retrospective cohort studies, six cross-sectional studies, one qualitative study, one randomized clinical trial, one systematic review, four literature reviews, and one practice guide. Our review shows that patients experienced different psychological phases after LVAD implantation. However, as the time from implantation progressed, these patients showed a significant improvement in depression, anxiety, and health-related quality of life.
\end{abstract}

Categories: Cardiology, Internal Medicine, Psychology

Keywords: depression in lvad patient, psychological stress in lvad patients, heart assist device and depression, heart assist device (psychology)

\section{Introduction And Background}

Over the years, heart failure has garnered multiple definitions, most of which explicitly define it as a clinical syndrome characterized by a variety of symptoms (shortness of breath, lower limb swelling, weakness) and signs (elevated jugular venous pressure, pulmonary congestion, peripheral swelling) caused by structural and functional cardiac abnormality resulting in reduced cardiac output and elevated intra-cardiac pressures [1]. Heart failure cases are estimated to rise to eight million by 2020 in the United States [2]. The disabling nature of heart failure is commonly associated with depression. The prevalence rate of depression in patients with heart failure is about $20 \%-40 \%$, which is $4 \%-5 \%$ higher for major depression in the general population [3]. Depression in patients with heart failure results in low quality of life, poor self-care, increased use of medical resources, and high readmission rate and mortality rate [4]. To enhance the outcome in advanced heart failure patients, who are unresponsive to medical management, left ventricular assist device (LVAD) has become a therapeutic choice. LVADs may serve as a bridge to heart transplant or destination therapy for poor transplant candidates. The goal for implanting an LVAD is to improve the overall quality of life of patients with advanced heart failure; studies have consistently shown that patients' quality of life is significantly enhanced post-LVAD implantation $[5,6]$. Okam et al. This is an open access Creative Commons Attribution License CC-BY 4.0., which permits unrestricted use, distribution, and reproduction in any medium, provided the original author and source are credited.

The symptoms of advanced heart failure can mask the symptoms of depression as they both have certain similarities (i.e., sleep disturbances, fatigue, or low energy). This can represent a diagnostic challenge in differentiating the typical symptoms of heart failure against those of depressed patients [7]. Multiple symptoms caused by heart failure can create a burden on the psychological health of patients. The mental stress these patients experience can easily remain undiagnosed due to their reluctance to disclose emotional stress out of fear of being labeled mentally ill; also, health professionals focus more on the treatment of heart failure symptoms and may write off depressive symptoms as a normal response to heart failure [8-12]. Ultimately, depression manifests itself through physical symptoms such as loss of appetite, weight loss, and sleep disturbance. Thus, most patients consult with other specialists rather than psychiatrists [8-12]. It is of utmost importance to identify heart failure patients with concomitant depression. Only after identifying heart failure patients with comorbid depression will we truly understand the role of LVAD in alleviating or worsening depression in patients with heart failure peri- and post-LVAD implantation. Some recipients of LVAD also describe emotional and psychological stress due to difficulties encountered in the care of the device (e.g., adapting to dependence on a power source and handling of the device), reduced sleep, pain, reduced activities of daily living, and reduced adherence to complex medication regimens [13]. The symptoms experienced by patients after LVAD implantation need to be thoroughly studied to understand if the resultant symptoms are an initial response to major surgery or heart failure depression. 


\section{Cureus}

This literature review will compare data from various studies providing an insight into understanding the overall rate of depression in patients who received LVADs, identify the psychological phases experienced by patients post-LVAD implantation, and whether LVAD improves existent heart failure depression or brings about a new-onset depression.

\section{Review}

Using parallel strategies to identify original research and review articles from PubMed, data was collected using Medical Subject Headings (MeSH) and regular keywords. After carefully reviewing titles, abstracts, and free full-text articles, relevant publications and their reference lists were reviewed to identify additional publications. Table 1 presents MeSH and regular keywords used for the literature search.

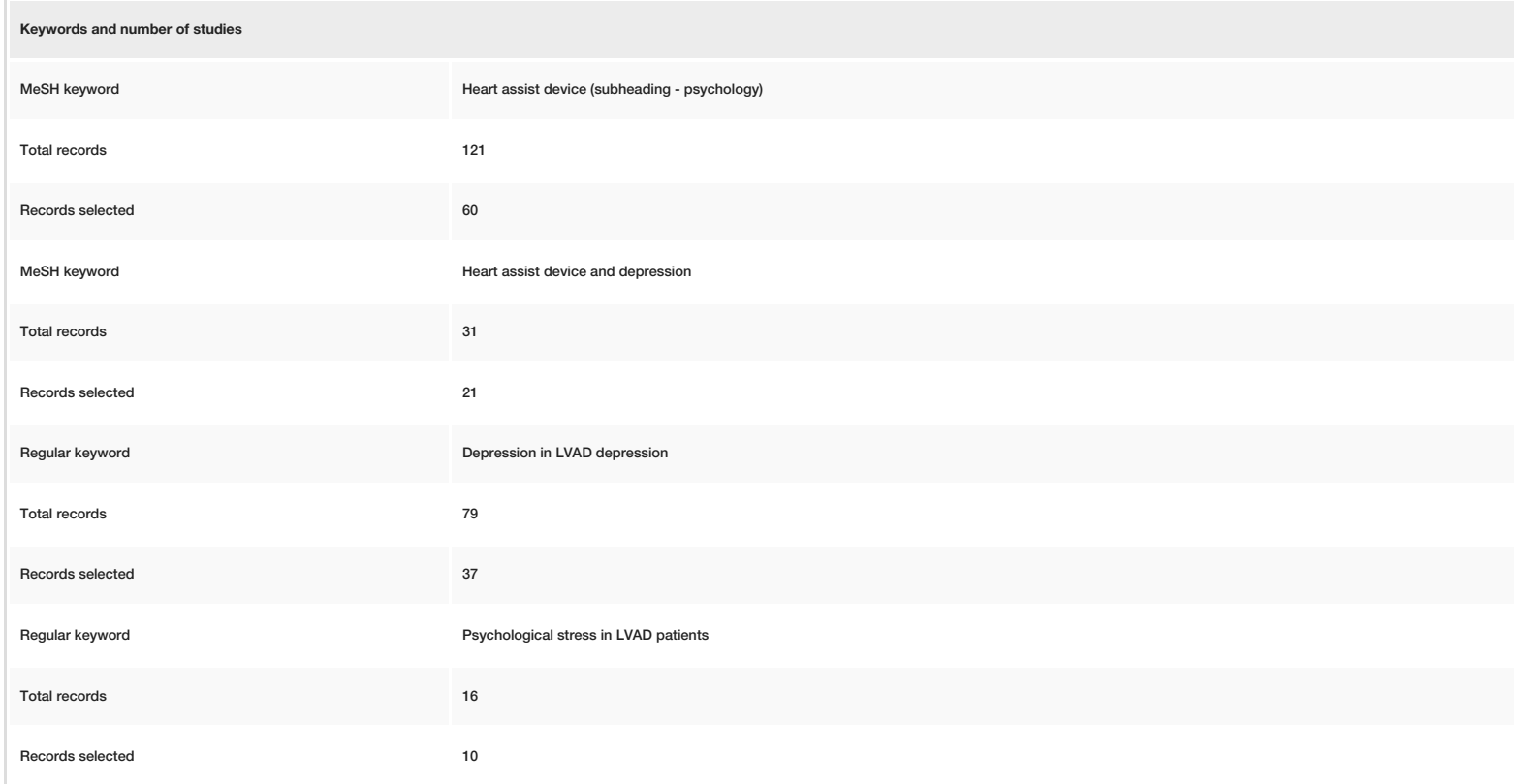

TABLE 1: MeSH and regular keywords used to find relevant publications appropriate for this literature review

LVAD, left ventricular assist device; MeSH, Medical Subject Headings

Studies were selected based on the following inclusion criteria: (1) English publications, (2) studies published within the past 10 years, (3) human subjects above 19 years old, (4) observational studies and clinical trials, including randomized controlled trials, cohort studies, case-control studies, or review articles. However, studies were exempted using the following exclusion criteria: (1) non-English language publications, (2) human subjects below 19 years old, (3) animal studies, (4) case series and meta-analyses. Table 2 presents the total number of studies selected after applying the inclusion/exclusion criteria. 


\section{Cureus}

\begin{tabular}{|c|c|}
\hline \multicolumn{2}{|c|}{ Number of studies after applying the inclusion/exclusion criteria } \\
\hline MeSH keyword & Heart assist device (subheading - psycholog \\
\hline Total records & 121 \\
\hline \multicolumn{2}{|l|}{ Inclusion/exclusion criteria } \\
\hline Published within 10 years & 86 \\
\hline Patient age $\geq 19$ years old & 61 \\
\hline Humans & 60 \\
\hline English publication & 60 \\
\hline MeSH keyword & Heart assist device and depression \\
\hline Total records & 31 \\
\hline \multicolumn{2}{|l|}{ Inclusion/exclusion criteria } \\
\hline Published within 10 years & 26 \\
\hline Patient age $\geq 19$ years old & 21 \\
\hline Humans & 21 \\
\hline English publication & 21 \\
\hline Regular keyword & Depression in LVAD patients \\
\hline Total records & 71 \\
\hline \multicolumn{2}{|l|}{ Inclusion/exclusion criteria } \\
\hline Publication within 10 years & 57 \\
\hline Patient age $\geq 19$ years old & 37 \\
\hline Humans & 37 \\
\hline English publication & 37 \\
\hline Regular keyword & Psychological stress in LVAD patients \\
\hline Total records & 16 \\
\hline \multicolumn{2}{|l|}{ Inclusion/exclusion } \\
\hline Publication within 10 years & 14 \\
\hline Patient age $\geq 19$ years old & 10 \\
\hline Humans & 10 \\
\hline English publication & 10 \\
\hline
\end{tabular}

TABLE 2: Total number of studies selected for the review after applying the inclusion/exclusion criteria

LVAD, left ventricular assist device; MeSH, Medical Subject Headings

Applying the regular and MeSH keywords using the inclusion and exclusion criteria, 118 articles were selected. After a thorough review of titles, abstracts, and full-text publications, 78 studies, due to the lack of information about the disease of interest (psychological stress in patients with LVAD implantation), article duplicates, case reports, and meta-analysis, were excluded. Finally, 40 publications in PubMed were selected, of which 27 publications were available in full text online and included the following:

- Twenty observational studies of which eight were prospective cohort studies, five retrospective cohort studies, six cross-sectional studies, and one qualitative study [12-31] 


\section{Cureus}

- One randomized clinical trial [32]

- One systematic review ( $\mathrm{n}=1887)[33]$

- Four literature reviews [7,34-36]

- One practice guideline [1].

Thirteen paid publications included mostly observational studies and literature reviews [37-49]. After reviewing 27 free available full-text articles, the minimum number of subjects in a study was nine, and the maximum was $1887[33,49]$. In total, the number of subjects in all publications excluding the review article was 4721 [12-33,38,39,41-45,48,49]. Among all 40 studies, 23 studies focused on LVADs' psychological implications in heart failure patients. Two publications also included the psychological experience of secondary individuals, such as spouses and caregivers of LVAD implantation patients explicitly [29,31]. All 27 publications are available for review in the free full-text form on PubMed and citations on information borrowed from the literature are provided. Figure 1 represents a flowchart of the selection process of the current literature review.

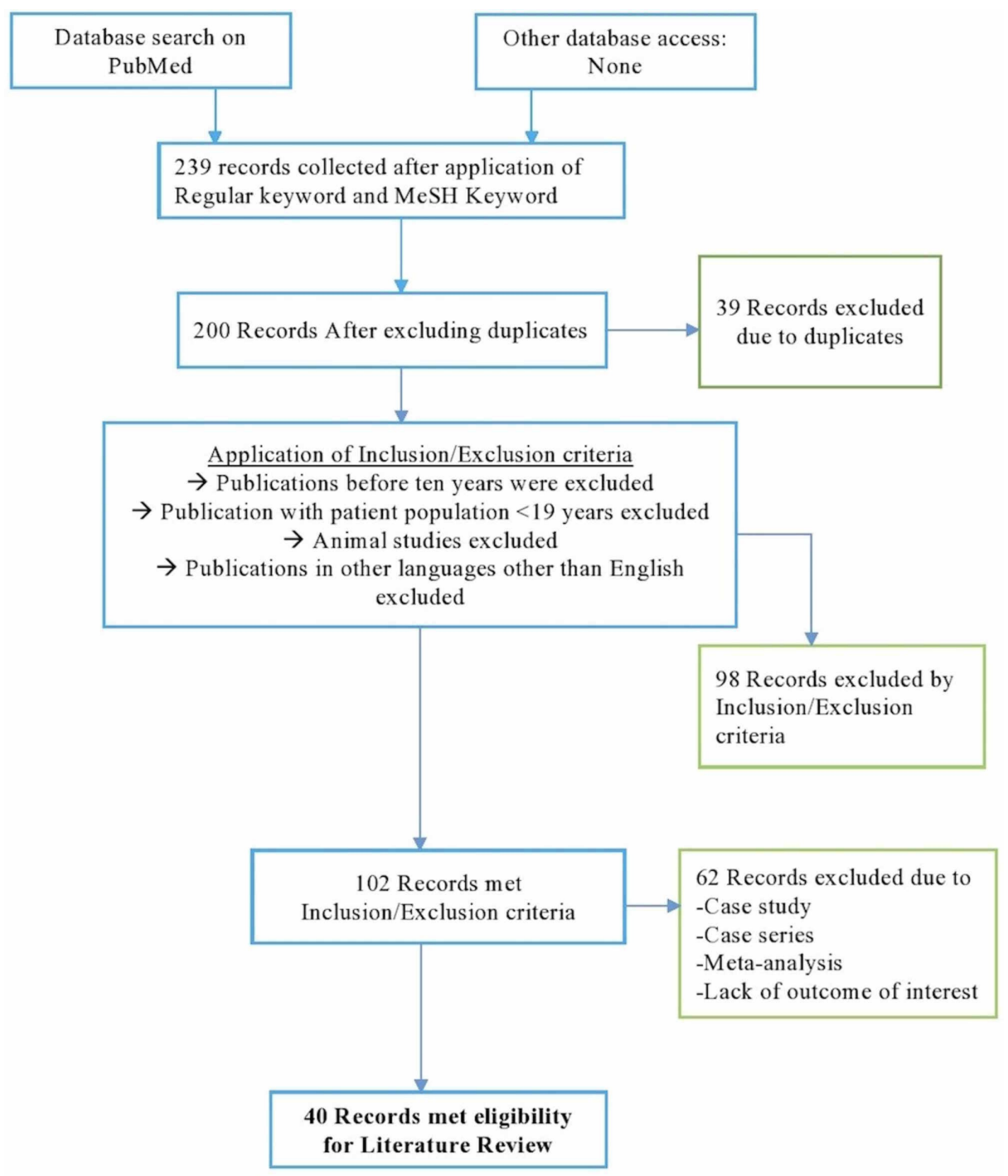

FIGURE 1: Flowchart representation of the selection process of the current literature review

MeSH, Medical Subject Headings

\section{Discussion}

The analysis conducted was intended to demonstrate the overall rate of depression in patients who received 
LVAD and identify the post-LVAD psychological phases experienced by these individuals. Advanced heart failure patients may eventually evolve from medical management to requiring a heart transplant. Due to the limited availability of donor heart or weak candidacy, some patients may require a heart assist device either as a bridge to heart transplant or as destination therapy. Most patients with advanced heart failure already experience anxiety, depression, and other mood disturbances, which if diagnosed early can improve outcomes after LVAD implantation. The evaluation of the psychological response LVAD patients experience after implantation has led to the synopsis of four phases. In the pre-LVAD period, patients provide an insight into the limitation brought on by heart failure symptoms and how the desperate need to relieve these symptoms may have influenced their decision on LVAD implantation [35]. The next phase after LVAD implantation is the hospitalization phase [35]. During this phase, patients are highly dependent on the medical team and learn essential skills about the heart device, which can be overwhelming for them [35]. Studies show a high prevalence of adjustment disorder after LVAD implantation [14,22]. During this phase, the anxiety level is amplified, which can be explained by the presence, weight, and sound of the heart assist device. In this phase, patients can also experience disruption in their endocrine, metabolic, cerebral circulation, or adverse effect of medication [14]. The third phase is an early adaptation phase [35]. In the early adaptation phase, patients learn to apply skills learned from the hospital to their everyday routine and modify their home environment to accommodate the electrical necessity of the heart assist device [35]. In this phase, patients are usually anxious as they recognize the need to be attentive as the heart assist device is vital in sustaining their lives [14]. Due to the vulnerability of patients in this phase, caregivers play a huge role in their physical and psychological well-being. Lastly, in the final stage described as a late adaptation phase, the patient becomes accepting of the new normal and show appreciation of the device [35]. In this stage, patients express a sense of increased self-esteem as they understand the complexities of the heart device and how to manipulate it [35]. The duration of these phases can vary as every patient responds differently to stressors.

After an overall view of the selected studies, majority of them showed statistically significant improvement in anxiety, depression, and quality of life after LVAD implantation with some studies identifying changes as early as within 30 days to 12 months after implantation regardless of treatment strategy (bridge to therapy vs. destination therapy). The ability of humans to adapt to changes can explain the findings in the studies. In the past 10 years, multiple research on patients with LVAD implantation has provided more insight into understanding patients' psychological responses to the implantation of a heart device designed to keep them alive. Tables 3, 4 summarize retrospective cohort studies, prospective cohort studies, and systematic reviews on the evolution of ideas on patients' psychological well-being post-LVAD implantation.

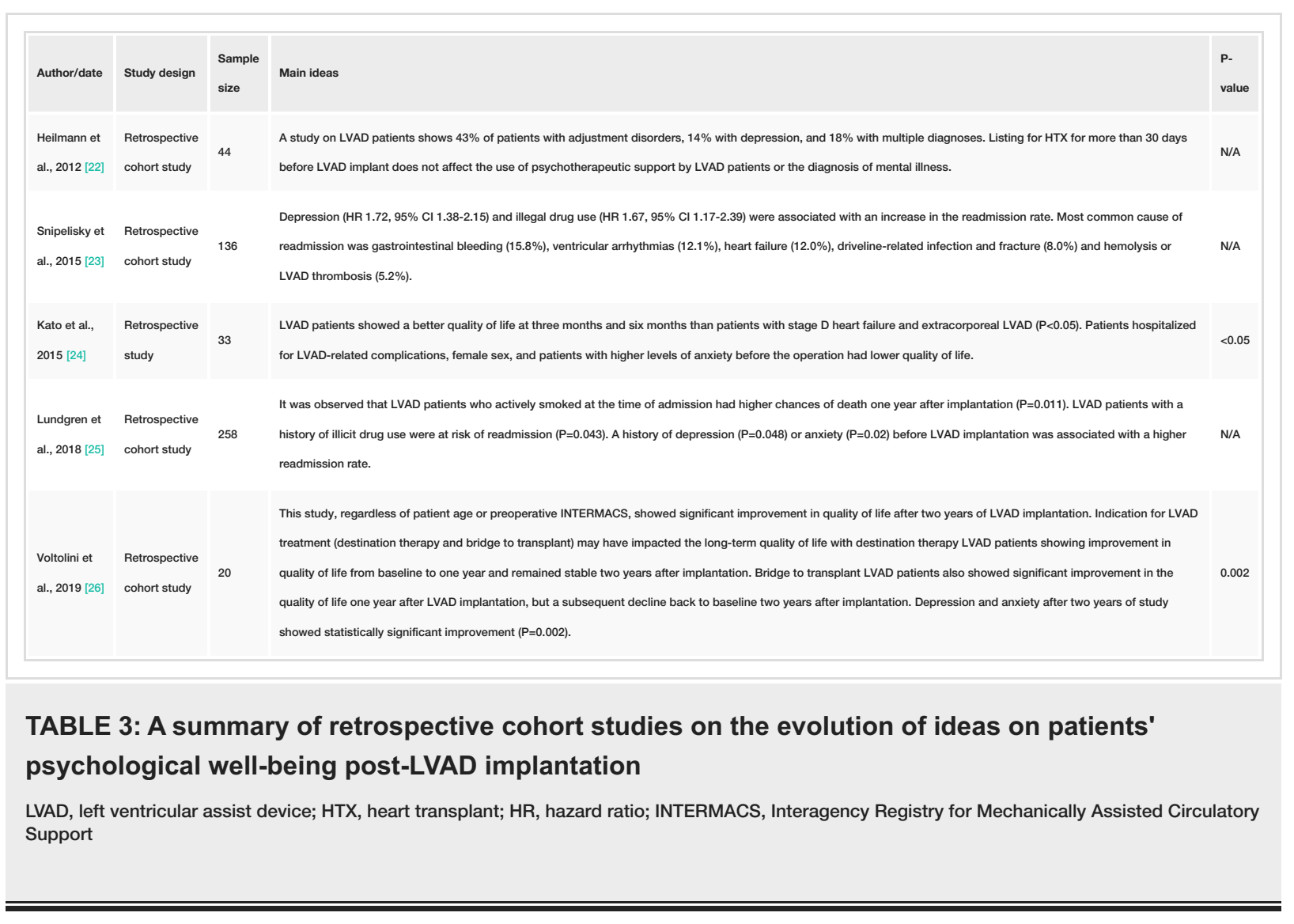




\section{Cureus}

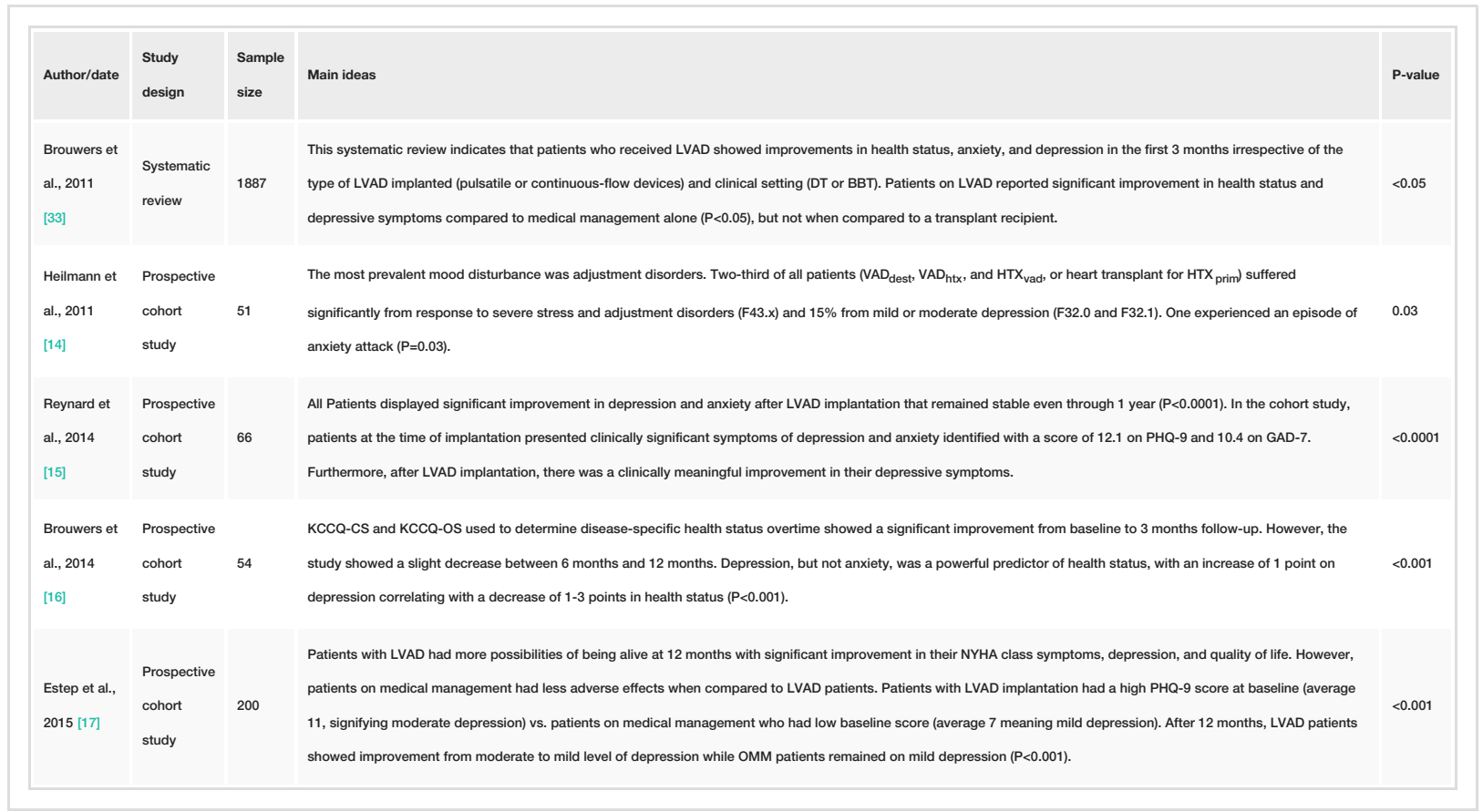

\section{TABLE 4: Prospective cohort studies and systematic reviews on the evolution of ideas on patients' psychological well-being after LVAD implantation}

LVAD, left ventricular assist device; DT, destination therapy; BTT, bridge to transplant; $\mathrm{HTX}_{\text {prim, }}$, primary heart transplantation; $\mathrm{VAD}_{\text {dest }}$, ventricular assist device on destination therapy; $\mathrm{VAD}_{\mathrm{htx}}$, ventricular assist device waiting for transplant; $\mathrm{HTX}_{\mathrm{vad}}$, successfully bridged to transplant on ventricular assist device; PHQ-9, Patient Health Questionnaire - 9; GAD-7, Generalized Anxiety Disorder 7-Item; KCCQ-CS, Kansas City Cardiomyopathy Questionnaire - Clinical Summary; KCCQ-OS, Kansas City Cardiomyopathy Questionnaire - Overall Summary; NYHA, New York Heart Association; OMM, on medical management

A randomized clinical trial compared the outcome between women and men using a continuous-flow LVAD as a bridge to transplantation. The study showed that the survival rate between men and women with LVAD was equivalent [32]. The percentage of patients with New York Heart Association (NYHA) functional class I or II symptoms improved from $0 \%$ at baseline to $83 \%$ for women and $85 \%$ for men at six months $(\mathrm{P}<0.001)$ [32].

Next are summaries of more prospective cohort studies that provided in-depth insight into the research analogy. Patient-Reported Outcomes Measurement Information System ${ }^{\circledR}$ (PROMIS ${ }^{\circledR}$ ) Depression sleep disturbance short form (SF) 8a and Anxiety sleep disturbance SF8a showed patients had a mean t-score of 56 $( \pm 5)$ and $53( \pm 12)$, respectively, before LVAD implantation. In contrast, the t-score changed to $44( \pm 6)$ and 42 $( \pm 9)$, respectively, 48 weeks after implantation. When plotted on a generalized linear mixed (GLM) model, a decrease overtime signifying improvement with $\mathrm{P}=0.03$ and $\mathrm{P}<0.001$ for depression and anxiety, respectively, was observed [18]. Another study by Shah et al. showed improvement in health-related quality of life (HRQOL), depression, and heart failure symptoms seen in LVAD patients as compared to patients on medical management in the Interagency Registry for Mechanically Assisted Circulatory Support (INTERMACS) 4 group [19]. The two-year survival of LVAD patients, when compared with optimal medical management (OMM) patients in INTERMACS 4 group met all-composite end-point with improvements in (1) New York Heart Association (NYHA) functional class ( $\geqslant 1$ class), (2) EuroQoL (EQ)-5D visual analog scale (VAS) $(>20$ points), and (3) Patient Health Questionnaire - 9 (PHQ-9) score ( $\geqslant 5$ points) $(\mathrm{P}<0.001)$ [19]. In a study by Lee et al., during the follow-up period of 6 months, LVAD patients in both patient groups (destination therapy and bridge to transplant) showed similar significant improvements in HRQOL, and significant improvement in shortness of breath, while moderate to significant improvements in wake disturbances [20]. A similar study, also conducted by Lee et al., showed significant improvement in shortness of breath (<0.001) and fatigue within 30 days after LVAD implantation, which was followed by subsequent improvement through 180 days [21]. During the study, it was found that heart failure causes the cardiac muscle to stretch, which led to the elevation of serum $\mathrm{N}$-terminal pro-B-type natriuretic peptide (NTproBNP) [21]. The implantation of LVAD led to a reduction in myocardial stretching and improvement in cardiac output. Thus, changes in myocardial stretching, which was measured by NTproBNP, were associated with an enhancement in shortness of breath, fatigue, cough, early satiety, and depression [21]. The findings in each study are crucial in the management of patients with LVAD implantation. It is essential to know the psychological implications exerted by the heart assist device in an individual's life, as a drastic change in one's routine can impact his or her social, physical, sexual, and psychological experience. The most extensive and most current study in our database had a follow-up period of two years after LVAD 
implantation [26]. Findings in this study are consistent with evidence that LVAD is highly effective in enhancing hemodynamics and improving functional capacity, which resulted in a favorable effect on the aspect of anxiety and depression in the two-year follow-up period. However, due to a lack of improvement in pain, discomfort, and self-care, patients experience difficulties with LVAD management and care, including LVAD tasks and limitations, and device's effect on self-image [26].

Despite a decrease in depression and anxiety in the majority of studies, adjustment disorders were found in $43 \%$, depression in $14 \%$, and multiple diagnoses in $18 \%$ LVAD patients [22]. Symptoms of depression and anxiety can be debilitating, leading to an increase in medication non-compliance, hospital readmission, higher medication cost, higher mortality rate, and decline in physical health [33]. Thus, early identification is necessary as the incorporation of psychological support as a significant part of their treatment plan might be lifesaving. It is clinically and statistically significant to assume that improvement in LVAD patients' mental well-being reflects on their quality of life as studies show depression in these patients to be a powerful predictor of their quality of life $[16,28,30]$. Partners of patients who received LVAD implantation reported higher levels of psychological breakdown, anxiety, and depression than LVAD patients [29]. They, therefore, stand a chance to gain from psychological therapy. An exciting find was in a study that measured saliva cortisol as a physiological response to stress in patients post-LVAD implantation [28]. This study shows the correlation between psychological and physiological factors in these patients as normal morning saliva cortisol was associated with low levels of depression in the study sample [28].

Limitations

There are limitations to writing a literature review on the psychological effect of LVAD implantation in heart failure patients. The variabilities of heart failure as a subject and relatively limited information on an LVAD can explain these limitations. The target population of choice posed a challenge. Only limited studies were available on patients who received LVAD, and already available reviews had additional variables in their analyses, which affected the literature's homogeneity. Current research studies restricted their review in terms of gender (no gender-specific research) and length of follow-up (no study discussed long-term followup beyond two years). Studies used various modalities and criteria to diagnose depression, anxiety, and other mood disorders in LVAD patients. As such, the analysis and comparison of the results of multiple studies presented a challenge.

\section{Conclusions}

The current literature concludes that in the peri-implantation and immediate post-LVAD implantation periods, patients experienced depression, anxiety, and mood disorders. However, as time elapsed, these patients passed through psychological phases of various lengths where they learned to adapt to changes, and shortly after, the psychological burden brought on by the heart assist device improved exponentially. This review should prompt researchers to conduct lengthier studies that are gender specific and explore more on the psychological phases experienced after LVAD implantation. Researches should provide knowledge on how medical management can influence each psychological phase and if medical intervention can shorten the duration of these phases and optimize LVAD patients' quality of life.

\section{Additional Information}

\section{Disclosures}

Conflicts of interest: In compliance with the ICMJE uniform disclosure form, all authors declare the following: Payment/services info: All authors have declared that no financial support was received from any organization for the submitted work. Financial relationships: All authors have declared that they have no financial relationships at present or within the previous three years with any organizations that might have an interest in the submitted work. Other relationships: All authors have declared that there are no other relationships or activities that could appear to have influenced the submitted work.

\section{References}

1. Ponikowski P, Voors AA, Anker SD, et al.: 2016 ESC Guidelines for the diagnosis and treatment of acute and chronic heart failure: the Task Force for the diagnosis and treatment of acute and chronic heart failure of the European Society of Cardiology (ESC). Developed with the special contribution of the Heart Failure Association (HFA) of the ESC. Eur Heart J. 2016, 37:2129-2200. 10.1093/eurhearti/ehw128

2. Go AS, Mozaffarian D, Roger VL, et al.: Heart disease and stroke statistics - 2014 update: a report from the American Heart Association. Circulation. 2014, 129:e28-e292. 10.1161/01.cir.0000441139.02102.80

3. Mbakwem A, Aina F, Amadi C: Expert opinion. Depression in patients with heart failure: is enough being done?. Card Fail Rev. 2016, 2:110-112. 10.15420/cfr.2016:21:1

4. Rustad JK, Stern TA, Hebert KA, Musselman DL: Diagnosis and treatment of depression in patients with congestive heart failure: a review of the literature. Prim Care Companion CNS Disord. 2013, 15:PCC.13r01511. 10.4088/PCC.13r01511

5. Gustafsson F, Rogers JG: Left ventricular assist device therapy in advanced heart failure: patient selection and outcomes. Eur J Heart Fail. 2017, 19:595-602. 10.1002/ejhf.779

6. Maciver J, Ross HJ: Quality of life and left ventricular assist device support . Circulation. 2012, 126:866-874. 10.1161/circulationaha.111.040279 
7. Celano CM, Villegas AC, Albanese AM, Gaggin HK, Huffman JC: Depression and anxiety in heart failure: a review. Harv Rev Psychiatry. 2018, 26:175-184. 10.1097/hrp.0000000000000162

8. Scherer M, Himmel W, Stanske B, Scherer F, Koschack J, Kochen MM, Herrmann-Lingen C: Psychological distress in primary care patients with heart failure: a longitudinal study. Br J Gen Pract. 2007, 57:801-807.

9. Saveanu RV, Mayes T: Diagnosing depression in congestive heart failure. Heart Fail Clin. 2011, 7:75-79. 10.1016/j.hfc.2010.08.010

10. Polikandrioti M: Needs of depressed patients with coronary artery disease . Health Sci J. 2011, 5:241-242.

11. Gnanasekaran G: Epidemiology of depression in heart failure. Heart Fail Clin. 2011, 7:1-10. 10.1016/j.hfc.2010.08.002

12. Polikandrioti M, Goudevenos J, Michalis LK, Koutelekos J, Kyristi H, Tzialas D, Elisaf M: Factors associated with depression and anxiety of hospitalized patients with heart failure. Hellenic J Cardiol. 2015, 56:26-35.

13. Abshire M, Russell SD, Davidson PM, et al.: Social support moderates the relationship between perceived stress and quality of life in patients with a left ventricular assist device. J Cardiovasc Nurs. 2018, 33:E1-E9. 10.1097/jcn.0000000000000487

14. Heilmann C, Kuijpers N, Beyersdorf F, et al.: Supportive psychotherapy for patients with heart transplantation or ventricular assist devices. Eur J Cardiothorac Surg. 2011, 39:e44-e50. 10.1016/j.ejcts.2010.11.074

15. Reynard AK, Butler RS, McKee MG, Starling RC, Gorodeski EZ: Frequency of depression and anxiety before and after insertion of a continuous flow left ventricular assist device. Am J Cardiol. 2014, 114:433-440. 10.1016/j.amjcard.2014.05.015

16. Brouwers C, de Jonge N, Caliskan K, et al.: Predictors of changes in health status between and within patients 12 months post left ventricular assist device implantation. Eur J Heart Fail. 2014, 16:566-573. 10.1002/ejhf.75

17. Estep JD, Starling RC, Horstmanshof DA, et al.: Risk assessment and comparative effectiveness of left ventricular assist device and medical management in ambulatory heart failure patients: results from the ROADMAP study. J Am Coll Cardiol. 2015, 66:1747-1761. 10.1016/j.jacc.2015.07.075

18. Weerahandi H, Goldstein N, Gelfman LP, et al.: The relationship between psychological symptoms and ventricular assist device implantation. J Pain Symptom Manage. 2017, 54:870-876.E1. 10.1016/j.jpainsymman.2017.05.002

19. Shah KB, Starling RC, Rogers JG, et al.: Left ventricular assist devices versus medical management in ambulatory heart failure patients: an analysis of INTERMACS Profiles 4 and 5 to 7 from the ROADMAP study. J Heart Lung Transplant. 2018, 37:706-714. 10.1016/j.healun.2017.12.003

20. Lee CS, Gelow JM, Chien CV, et al.: Implant strategy-specific changes in symptoms in response to left ventricular assist devices. J Cardiovasc Nurs. 2018, 33:144-151. 10.1097/jcn.0000000000000430

21. Lee CS, Mudd JO, Lyons KS, et al.: Heart failure symptom biology in response to ventricular assist device implantation. J Cardiovasc Nurs. 2019, 34:174-182. 10.1097/jcn.0000000000000552

22. Heilmann C, Kuijpers N, Beyersdorf F, et al.: Does listing for heart transplant for longer than 30 days before ventricular assist device implantation influence utilization of psychotherapeutic support and outcome?. Eur J Cardiothorac Surg. 2012, 41:1371-1376. 10.1093/ejcts/ezr233

23. Snipelisky D, Stulak JM, Schettle SD, Sharma S, Kushwaha SS, Dunlay SM: Psychosocial characteristics and outcomes in patients with left ventricular assist device implanted as destination therapy. Am Heart J. 2015, 170:887-894. 10.1016/j.ahj.2015.08.012

24. Kato NP, Okada I, Imamura T, et al.: Quality of life and influential factors in patients implanted with a left ventricular assist device. Circ J. 2015, 79:2186-2192. 10.1253/circj.CJ-15-0502

25. Lundgren S, Lowes BD, Zolty R, Burdorf A, Raichlin E, Um JY, Poon C: Do psychosocial factors have any impact on outcomes after left ventricular assist device implantation?. ASAIO J. 2018, 64:e43-e47. 10.1097/mat.0000000000000736

26. Voltolini A, Salvato G, Frigerio M, Cipriani M, Perna E, Pisu M, Mazza U: Psychological outcomes of left ventricular assist device long-term treatment: a 2-year follow-up study. Artif Organs. 2020, 44:67-71. 10.1111/aor.13531

27. Heilmann C, Kaps J, Hartmann A, et al.: Mental health status of patients with mechanical aortic valves, with ventricular assist devices and after heart transplantation. Interact Cardiovasc Thorac Surg. 2016, 23:321325. 10.1093/icvts/ivw111

28. Abshire M, Bidwell JT, Page G, et al.: Physiological and psychological stress in patients living with a left ventricular assist device. ASAIO J. 2018, 64:172-180. 10.1097/mat.0000000000000847

29. Kugler C, Meng M, Rehn E, Morshuis M, Gummert JF, Tigges-Limmer K: Sexual activity in patients with left ventricular assist devices and their partners: impact of the device on quality of life, anxiety and depression. Eur J Cardiothorac Surg. 2018, 53:799-806. 10.1093/ejcts/ezx426

30. Casida JM, Abshire M, Ghosh B, Yang JJ: The relationship of anxiety, depression, and quality of life in adults with left ventricular assist devices. ASAIO J. 2018, 64:515-520. 10.1097/mat.0000000000000681

31. Wilhelms LA, Blumenthal-Barby JS, Kostick KM, Estep JD, Bruce CR: Patients' perspectives on transplantation while undergoing left ventricular assist device support. ASAIO J. 2017, 63:740-744. 10.1097/mat.0000000000000567

32. Bogaev RC, Pamboukian SV, Moore SA, et al.: Comparison of outcomes in women versus men using a continuous-flow left ventricular assist device as a bridge to transplantation. J Heart Lung Transplant. 2011, 30:515-522. 10.1016/j.healun.2010.12.009

33. Brouwers C, Denollet J, de Jonge N, Caliskan K, Kealy J, Pedersen SS: Patient-reported outcomes in left ventricular assist device therapy: a systematic review and recommendations for clinical research and practice. Circ Heart Fail. 2011, 4:714-723. 10.1161/circheartfailure.111.962472

34. Grogan S, Kostick K, Delgado E, Bruce CR: Ventricular assist devices as destination therapy: psychosocial and ethical implications. Methodist Debakey Cardiovasc J. 2015, 11:9-11. 10.14797/mdcj-11-1-9

35. Abshire M, Prichard R, Cajita M, DiGiacomo M, Dennison Himmelfarb C: Adaptation and coping in patients living with an LVAD: a metasynthesis. Heart Lung. 2016, 45:397-405. 10.1016/j.hrtlng.2016.05.035

36. Bordoni B, Marelli F, Morabito B, Sacconi B: Depression and anxiety in patients with chronic heart failure . 
Future Cardiol. 2018, 14:115-119. 10.2217/fca-2017-0073

37. Maciver J, Rao V, Ross HJ: Quality of life for patients supported on a left ventricular assist device . Expert Rev Med Devices. 2011, 8:325-337. 10.1586/erd.11.9

38. Casida JM, Brewer RJ, Smith C, Davis JE: An exploratory study of sleep quality, daytime function, and quality of life in patients with mechanical circulatory support. Int J Artif Organs. 2012, 35:531-537. 10.5301/ijao.5000109

39. Overgaard D, Grufstedt Kjeldgaard H, Egerod I: Life in transition: a qualitative study of the illness experience and vocational adjustment of patients with left ventricular assist device. J Cardiovasc Nurs. 2012, 27:394-402. 10.1097/JCN.0b013e318227f119

40. Bruce CR, Delgado E, Kostick K, et al.: Ventricular assist devices: a review of psychosocial risk factors and their impact on outcomes. J Card Fail. 2014, 20:996-1003. 10.1016/j.cardfail.2014.09.006

41. Sandau KE, Hoglund BA, Weaver CE, Boisjolie C, Feldman D: A conceptual definition of quality of life with a left ventricular assist device: results from a qualitative study. Heart Lung. 2014, 43:32-40. 10.1016/j.hrtlng.2013.09.004

42. Modica M, Ferratini M, Torri A, Oliva F, Martinelli L, De Maria R, Frigerio M: Quality of life and emotional distress early after left ventricular assist device implant: a mixed-method study. Artif Organs. 2015, 39:220227. 10.1111/aor.12362

43. Brouwers C, Denollet J, Caliskan K, et al.: Psychological distress in patients with a left ventricular assist device and their partners: an exploratory study. Eur J Cardiovasc Nurs. 2015, 14:53-62. $10.1177 / 1474515113517607$

44. Casida JM, Wu HS, Abshire M, Ghosh B, Yang JJ: Cognition and adherence are self-management factors predicting the quality of life of adults living with a left ventricular assist device. J Heart Lung Transplant. 2017, 36:325-330. 10.1016/j.healun.2016.08.023

45. Yost G, Bhat G, Mahoney E, Tatooles A: Reduced anxiety and depression in patients with advanced heart failure after left ventricular assist device implantation. Psychosomatics. 2017, 58:406-414. 10.1016/j.psym.2017.02.001

46. Dornelas EA, Sears SF: Living with heart despite recurrent challenges: psychological care for adults with advanced cardiac disease. Am Psychol. 2018, 73:1007-1018. 10.1037/amp0000318

47. Adams EE, Wrightson ML: Quality of life with an LVAD: a misunderstood concept . Heart Lung. 2018, 47:177-183. 10.1016/j.hrtlng.2018.02.003

48. Modica M, Minotti A, De Maria R, et al.: Coping, mood, quality of life, and outcomes in recipients of left ventricular assist devices: a cluster analysis. Psychosom Med. 2019, 81:192-199. 10.1097/psy.0000000000000658

49. Casida JM, Marcuccilli L, Peters RM, Wright S: Lifestyle adjustments of adults with long-term implantable left ventricular assist devices: a phenomenologic inquiry. Heart Lung. 2011, 40:511-520. 10.1016/j.hrtlng.2011.05.002 